\title{
Cerebellar contribution to feedforward control of locomotion
}

\author{
Iolanda Pisotta and Marco Molinari* \\ Neurological Rehabilitation Department A and CaRMA Lab, I.R.C.C.S. Fondazione Santa Lucia Rome, Rome, Italy
}

Edited by:

Nadia Dominici, VU University

Amsterdam, Netherlands

Reviewed by:

Masao Ito, RIKEN, Japan

Richard E. Poppele, University of

Minnesota, USA

${ }^{*}$ Correspondence:

Marco Molinari, Neurological

Rehabilitation Department A and

CaRMA Lab, I.R.C.C.S. Fondazione

Santa Lucia Rome, Via Ardeatina

306, 00179 Rome, Italy

e-mail:m.molinari@hsantalucia.it
The cerebellum is an important contributor to feedforward control mechanisms of the central nervous system, and sequencing-the process that allows spatial and temporal relationships between events to be recognized-has been implicated as the fundamental cerebellar mode of operation. By adopting such a mode and because cerebellar activity patterns are sensitive to a variety of sensorimotor-related tasks, the cerebellum is believed to support motor and cognitive functions that are encoded in the frontal and parietal lobes of the cerebral cortex. In this model, the cerebellum is hypothesized to make predictions about the consequences of a motor or cognitive command that originates from the cortex to prepare the entire system to cope with ongoing changes. In this framework, cerebellar predictive mechanisms for locomotion are addressed, focusing on sensorial and motoric sequencing. The hypothesis that sequence recognition is the mechanism by which the cerebellum functions in gait control is presented and discussed.

Keywords: locomotion, corticocerebellar loops, feedforward control, movement prediction, sequencing hypothesis

\section{INTRODUCTION}

Based on animals and humans studies, much has been learned about how the cerebellum coordinates normal movement and how it contributes to motor adaptation and motor learning.

Cerebellar damage does not cause a loss of movement; instead, it effects clear and consistent abnormalities in movement, including lack of coordination, increased variability, tremor, and poor accuracy. Notably, cerebellar damage induces greater impairments to movements that require predictive control versus those that require reactive control. As demonstrated by Morton and Bastian (2006) using an elegant task that was based on splitbelt treadmill walking, cerebellar damage impairs the ability to adapt to predictable but not sudden unpredictable changes. Recently developed functional theories on predictive control of the cerebellum explain the effects of cerebellar damage on eye and limb movements and on walking (Koziol et al., 2014).

In motor control theories, the term "predictive" refers to the feedforward component of a movement that is planned in advance and is unchanged by online peripheral feedback. Predictive control is typically assessed at the earliest stage of the movement, during which corrections that are based on peripheral feedback are not possible. This type of control is used to make any online corrections that might be necessary as a movement unfolds, and it requires that the conditions of later movement stages be known in advance. Although the hypothesis that the cerebellum is important for predictive control is not new, claims of its importance in locomotive control are relatively recent (Morton and Bastian, 2006). Further, current data on the significance of cerebellar predictive control in areas outside of the motor domain and for supporting learning and functional recovery have piqued the interest of neuroscientists in the hopes of better understanding cerebellar control mechanisms.

In this framework, we will discuss cerebellar predictive mechanisms for locomotion, focusing on the type of information that is processed - sensorial or motoric - and on sequence recognition as the mechanisms for understanding cerebellar function in making predictions.

\section{CEREBELLUM AND FEEDFORWARD CONTROL}

The cerebellum has an exquisitely simple cellular organization, which has been well known since the beginning of the last century, based on the work of Ramon y Cajal (see Sotelo, 2008). Since then, scientists have been intrigued by its function. Nevertheless, after more than a century of dedicated studies, there is no consensus on how the cerebellum operates. Among the various theories that exist, the hypothesis that the cerebellum mediates predictive motor control in locomotion is gaining momentum (Bastian, 2006).

The forward model of motor control postulates that to make a motor-to-somatosensory prediction, the cerebellum receives an efference copy of a motor command from the primary motor cortex. This information allows the cerebellum to make a prediction with regard to the sensory consequences of such motor commands, allowing the musculoskeletal system to prepare to successfully execute a movement. During movement, predicted sensations are then compared with the actual incoming sensations. If there is a positive match, the pattern is maintained for the next movement. The lack of a match is associated with an alert signal that is sent back to the motor cortical and subcortical areas, which activates feedback movement corrections and calibration of the forward model (Shadmehr et al., 2010). 
This process allows the corticocerebellar circuit to act as somatic event detectors that respond, particularly to unexpected stimuli (Restuccia et al., 2007). Various studies on the internal forward model have demonstrated that the cerebellum generates motorto-somatosensory predictions (Izawa et al., 2012; Popa et al., 2012; Knolle et al., 2013).

Thus, consensus is building that the cerebellum is more involved in learning to associate motor commands with novel sensory consequences-i.e., the forward model-than in learning to correlate sensory goals with novel motor commands (the inverse model).

\section{SEOUENCING AND PREDICTION}

In 1997, Braitenberg, Heck, and Sultan proposed sequence detection and generation as the basic operational mode of the cerebellum in the motor domain (Braitenberg et al., 1997). Since then, the sequencing properties of cerebellar processing have been studied extensively, and sequencing has been reported to be the more frequently impaired function in a large cohort of subjects with cerebellar damage (Tedesco et al., 2011).

Few years ago, we proposed to consider sequencing the basic mechanism that allows cerebellar prediction in all functional domains (Molinari et al., 2008). Traditionally, sequencing has not been recognized as a discrete cognitive function, and it can be defined as a supramodal function, the relationships with other functions of which, such as working memory and timing, remain unknown. Acquiring and acting on a serial order of events is a fundamental ability that effects sequencing structure knowledge. To recognize that stimuli are presented in a particular order, sensory information that pertains to a stimulus must be kept active in a working memory system and compared with subsequent stimuli. Like many instrumental abilities, sequence knowledge can be acquired incidentally through experience (implicit learning) or intentionally through explicit effort (declarative learning). A schematic of cerebellar sequence mechanisms for prediction is shown in Figure 1.

Whenever feedforward control is needed, the cerebellum intervenes by identifying predictable patterns of motor or cognitive command sequences and linking them with learned sensory or cognitive consequences. This process allows anticipatory responses to be generated in all relevant cerebellar domains.

Cerebellar input has a facilitating effect on the contralateral cerebral cortex, and chronic cerebellar damage (Di Lazzaro et al., 1994) and cerebellar conditioning transcranial magnetic stimulation (TMS; Grimaldi et al., 2014) reduce the excitability of the contralateral motor cortex. Furthermore, the cerebellar influence on the cerebral cortex is not limited to motor areas, and the nature and functional significance of the overall cerebellar influence over the cerebral cortex is the subject of much debate (Dalal et al., 2013). At least for the motor domain, it is widely accepted that cerebellar input conveys information for sensory motor integration.

TMS experiments in rats support this model (Ben Taib et al., 2005; Oulad Ben Taib and Manto, 2008). In rats, as in humans, the enhancement of excitability in the contralateral motor cortex after sustained somatosensory stimulation is cerebellum-dependent (Kaelin-Lang et al., 2002; Luft et al.,

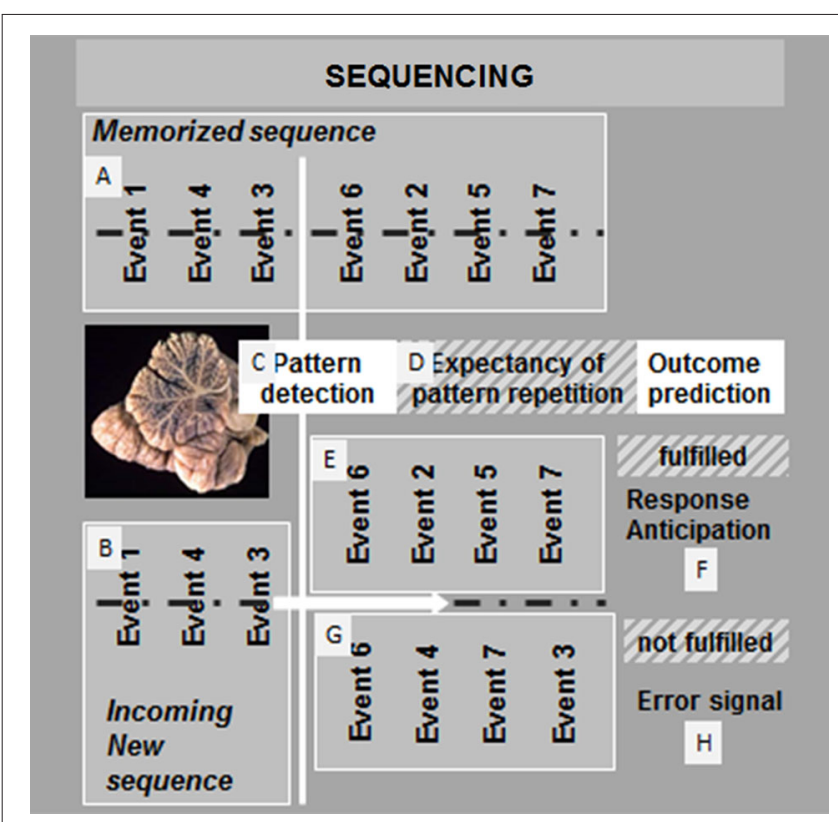

FIGURE 1 | Proposed mechanism of cerebellar sequencing for prediction. Incoming events are continuously monitored in the cerebellar circuits. Relations between events are compared in the cerebellar corticonuclear microcomplex (Ito, 2005) and stored in a working memory buffer (A). Through the same mechanisms, sequences of new incoming events are compared with previously stored event sequences (B). If a match is recognized (C), then an expectancy of repetition is generated (D). The cerebellar monitoring of the flow of events continues, and as long as the prediction is maintained (E), response anticipation is conveyed, and feedforward control can function smoothly (F). If prediction fails (G), then an error signal is activated by the cerebellar output system $\mathbf{( H )}$, and feedforward control is interrupted or corrected.

2002). Cerebellum-dependent neurophysiological changes are not limited to the motor cortex, and cerebellar output might also directly affect the somatosensory cortex. The parietal cortex projects to the cerebellum through the pontine nuclei in a topographically organized manner (Schmahmann and Pandya, 1989), and it receives the cerebellar return loop through the thalamus (Giannetti and Molinari, 2002; Allen et al., 2005; Clower et al., 2005). Cerebellar influences on the parietal somatosensory cortex have been demonstrated in cats (Kolodziejak et al., 2000) and in patients with unilateral cerebellar lesions (Restuccia et al., 2001). In subjects with unilateral cerebellar damage, late $\mathrm{N} 24$ and P24 components of the somatosensory-evoked potentials decline significantly in the contralateral somatosensory cortex (Restuccia et al., 2001). A magnetoencephalography (MEG) study that compared expected and unexpected sensory stimuli in evoking cerebellar and cortical responses has linked the somatosensonsory evoked potentials findings with the prediction and sequencing theories of cerebellar function. A regular train of somatosensory stimuli induces evoked potentials in the contralateral somatosensory cortex and ipsilateral cerebellum. If the stimulus is omitted at random, while no activity is recorded in the somatosensory cortex, cerebellar activity is markedly enhanced (Tesche and Karhu, 2000). This response after an unpredictable omission in a predictable sequence has been interpreted as 
proof of the ability of the cerebellum to code expectancy (Ivry, 2000).

Clinical evidence of cerebellar function in coding expectancy has been confirmed by 2 groups, both of which used mismatch negativity protocols. Restuccia et al. (2007) used a somatosensory mismatch negativity (S-MMN) protocol in patients with unilateral cerebellar lesions. S-MMN is believed to be generated by differences between current and prior inputs, supported by an automatic change-detection cortical process. This process is blocked or impaired if the cerebellar input is absent. Subjects who are affected by unilateral hemispheric cerebellar stroke do not develop S-MMN responses in the contralateral cortex. Similar findings have been observed in an MMN auditory paradigm (Moberget et al., 2008), focusing on timing expectancy. Both studies have demonstrated that the cerebellum is part of the MMN circuit and that it is critical for generating expectancy and making sensory predictions.

These findings complement the theories on cerebellar function in the prediction of sensory events (Nixon, 2003) and the longstanding hypothesis that the cerebellum acts as a comparator (Ito, 2005). In this theoretical framework, it is conceivable that through a comparison of time and space characteristics of actual and preceding stimuli, predictable event sequences can be recognized and stored. Thus, sequencing in the sensorimotor domain is evident, but is this also true when cognitive functions are considered?

Behavioral or script sequencing can be defined as the process that allows spatial and temporal relations to be recognized correctly among behaviorally relevant actions (Sirigu et al., 1998). Script sequencing is altered in subjects with cerebellar damage who are tested in an ad hoc card sorting task (Leggio et al., 2011) and is interpreted as a prediction deficit in the cognitive/behavioral domain. Card sequencing tasks require visual or verbal material to be examined to understand spatial, temporal, and/or semantic relationships and correctly reconstruct the strings in logical sequences.

The test in Leggio et al. (2008) consisted of 11 sets of cards, each comprising 6 cartoon-like drawings, including sentences (to examine verbal factors), behavioral figures (for behavioral factors), and abstract figures (for spatial factors), that were to be ordered in a logical sequence by patients. The influence of the lesion was analyzed by grouping patients by lesion type (focal or atrophic) and lesion side (right or left). Patients with cerebellar damage developed cognitive sequencing impairments, and lesion side and characteristics of the material that were to be sequenced correlated. Specifically, patients with left lesions performed poorly only on script sequences that were based on pictorial material, and patients with right lesions encountered difficulties with script sequences that required verbal elaboration. The presence of right/left and pictorial/verbal differences is consistent with the hypothesis that cerebrocerebellar interactions are organized in segregated corticocerebellar loops, in which specificity is related to the characteristics of the information that is processed-not to the mode of function (Leggio et al., 2008).

\section{CEREBELLAR GAIT}

Locomotion can be considered a purposeful, goal-directed behavior that is initiated by signals that arise from volitional processing in the cerebral cortex or emotional processing in the limbic system and sustained by basic locomotor motor patterns that are generated by spinal interneuronal networks-i.e., the central pattern generator (CPG) circuits. Locomotor control mechanisms are complex and, in addition to the CPGs, involve various subcortical and cortical control areas (for review, see Takakusaki, 2013). In this network, the cerebellum is considered dispensable for steadystate locomotion but crucial for avoiding obstacles and adapting to novel conditions.

Cerebellar gait ataxia is characterized by staggering, irregular stepping, veering, and excessive high lifting of the feet above the ground. This clinical condition has been linked to the inability to control relative movements between leg joints during locomotion. Starting from clinical observation, the coordination of multijoint activity through the scaling of movement variables has been considered the core of cerebellar motor function (Topka et al., 1998). Of the movement variables that are cerebellum-dependent, the timing of muscle activity, especially of antagonist muscles, has long been favored (Frysinger et al., 1984).

In 2001, Earhart and Bastian questioned cerebellar function in the timing or scaling of individual joint movements during gait by asking subjects with cerebellar damage to step on a surface that was inclined at various angles while walking. Healthy subjects mastered the task by using several temporal strategies, with systematic shifts in the timing of muscle activity and peak joint angles, based on the changes in inclination. Notably, cerebellar subjects were able to produce appropriate timing shifts at most joints, demonstrating preserved selection of the basic timing of motor patterns. Conversely, the presence of abnormal relative joint movements and the decomposition of movement implicated the cerebellum in adjusting the relative movement of multiple joints, especially to accommodate external constraints (Earhart and Bastian, 2001). Collectively, animal studies and clinical evidence have demonstrated cerebellar function in adaptive gait control, effecting constant recalibration of walking patterns to navigate various terrains and environments smoothly.

Cerebellar adaptation is not based on sensory feedback information. Subjects with cerebellar damage are impaired in locomotor tasks that require prediction, whereas they have good control when reactive control is needed (Morton and Bastian, 2006). As discussed, one possible mechanism of sustaining prediction is sequencing, which can intervene at various levels of locomotor control. Thus, similar to what has been observed in sMMN paradigms (Restuccia et al., 2007), fixed sequences of sensory information, funneled by spinocerebellar fibers during locomotion (Jankowska et al., 2011), have been hypothesized to be recognized by the cerebellum, effecting correct prediction of the neuromuscular requirements of the subsequent step. Alterations in the predicted sequence will enhance the cerebellar output system, allowing cortical and brainstem locomotor regions to adapt.

Conversely, subjects with cerebellar atrophies are not only impaired in managing obstacles and adapting to novel environment, they develop ataxic gait in well-learned environments and on smooth surfaces (Mari et al., 2014), implicating cerebellar 
processing in controlling steady-state locomotion. This clinical profile also exists in subjects with cerebellar stroke; nevertheless, gait ataxia is generally mild, from which patients recover well (Bultmann et al., 2014). Pascual-Leone and colleagues (Farzan et al., 2013) recently reported that 21 days of cerebellar TMS reduces gait ataxia in patients who are affected by idiopathic lateonset cerebellar atrophy. This finding suggests that low-frequency TMS reduces the inhibitory control of the cerebellar cortex over the dentate nucleus, favoring the function of dentate nucleus output.

To this end, we would like to advance an alternative hypothesis. Considering the established function role of motor learning and adaptation in allowing forward control strategies to be generated and the lack of cerebellar influence on reactive adjustments and well-learned automatic movements, it is conceivable that cerebellar TMS inhibits the cerebellar output, allowing motor circuits to act in the absence of cerebellar influences. Thus, the experimental condition that was proposed by Pasqual-Leone and colleagues could approximate a cerebellar focal lesion-i.e., after a stroke. Both conditions are associated with better locomotion than in the presence of cerebellar atrophy.

It follows that cerebellar gait ataxia due to cerebellar atrophy might be the result of erroneous cerebellar predictions. Altered cerebellar processing will insert virtual errors into the forward control models, inducing continuous correction of the ongoing motor command. If this hypothesis is true, the inhibition of cerebellar processing —e.g., by TMS or tDCS—would improve gait in subjects with cerebellar ataxia but will have little or no effect on subjects with ataxia due to focal cerebellar damage. Conversely, focal damage to the cerebellum is associated with balance and gait problems, primarily in the acute/subacute phase followed by efficient spontaneous functional recovery (Bultmann et al., 2014). This evidence suggests that the motor system recovers more efficiently from the absence of cerebellar processing than from alterations to it. This view is supported by recent literature on cerebellar stimulation (Farzan et al., 2013; Ferrucci et al., 2013; Grimaldi et al., 2014), suggesting that transcranial cerebellar stimulation is a feasible neurorehabilitation intervention that can be used to treat gait ataxia (Block and Celnik, 2012).

\section{CONCLUSION}

The importance of feedforward control in motor control and the significance of cerebellar processing in this function are well established. Nevertheless, in locomotion control, cerebellar function is neglected, and studies have focused primarily on spinal and cortical locomotion control mechanisms. Current evidence implicates forward models as more important locomotion control mechanisms, but the relative importance of forward and inverse models to locomotion remains unknown. Recent reports indicates that cerebellar processing intervenes in locomotion by providing advance information on subsequent step events, suggesting how such motor prediction can be obtained per the sequencing hypothesis of cerebellar function. In nearly all cerebellar functional domains-from motor to cognition-cerebellar symptoms can be attributed to impairments in recognizing repeated sequenced patterns. Only recognition of a previously experienced pattern allows a prediction to be made and thus effective feed-forward control to be instigated. This theory of cerebellar function has implications for the symptomatic treatment of gait disturbances, and preliminary results on magnetic and electrical modulation of cerebellar function are guiding the development of an effective treatment for ataxic gait.

\section{REFERENCES}

Allen, G., McColl, R., Barnard, H., Ringe, W. K., Fleckenstein, J., and Cullum, C. M. (2005). Magnetic resonance imaging of cerebellar-prefrontal and cerebellar-parietal functional connectivity. Neuroimage 28, 39-48. doi: 10 . 1016/j.neuroimage.2005.06.013

Bastian, A. J. (2006). Learning to predict the future: the cerebellum adapts feedforward movement control. Curr. Opin. Neurobiol. 16, 645-649. doi: 10.1016/j. conb.2006.08.016

Ben Taib, N. O., Manto, M., Pandolfo, M., and Brotchi, J. (2005). Hemicerebellectomy blocks the enhancement of cortical motor output associated with repetitive somatosensory stimulation in the rat. J. Physiol. 567(Pt. 1), 293-300. doi: 10. 1113/jphysiol.2005.088229

Block, H. J., and Celnik, P. (2012). Can cerebellar transcranial direct current stimulation become a valuable neurorehabilitation intervention? Expert Rev. Neurother. 12, 1275-1277. doi: 10.1586/ern.12.121

Braitenberg, V., Heck, D., and Sultan, F. (1997). The detection and generation of sequences as a key to cerebellar function: experiments and theory. Behav. Brain Sci. 20, 229-245; discussion 245-277. doi: 10.1017/s0140525x9721143x

Bultmann, U., Pierscianek, D., Gizewski, E. R., Schoch, B., Fritsche, N., Timmann, D., et al. (2014). Functional recovery and rehabilitation of postural impairment and gait ataxia in patients with acute cerebellar stroke. Gait Posture 39, 563-569. doi: 10.1016/j.gaitpost.2013.09.011

Clower, D. M., Dum, R. P., and Strick, P. L. (2005). Basal ganglia and cerebellar inputs to 'AIP'. Cereb. Cortex 15, 913-920. doi: 10.1093/cercor/ bhh190

Dalal, S. S., Osipova, D., Bertrand, O., and Jerbi, K. (2013). Oscillatory activity of the human cerebellum: the intracranial electrocerebellogram revisited. Neurosci. Biobehav. Rev. 37, 585-593. doi: 10.1016/j.neubiorev.2013.02.006

Di Lazzaro, V., Restuccia, D., Molinari, M., Leggio, M. G., Nardone, R., Fogli, D., et al. (1994). Excitability of the motor cortex to magnetic stimulation in patients with cerebellar lesions. J. Neurol. Neurosurg. Psychiatry 57, 108-110. doi: 10. 1136/jnnp.57.1.108

Earhart, G. M., and Bastian, A. J. (2001). Selection and coordination of human locomotor forms following cerebellar damage. J. Neurophysiol. 85, 759-769.

Farzan, F., Wu, Y., Manor, B., Anastasio, E., Lough, M., Novak, V., et al. (2013). Cerebellar TMS in treatment of a patient with cerebellar ataxia: evidence from clinical, biomechanics and neurophysiological assessments. Cerebellum 12, $707-$ 712. doi: 10.1007/s12311-013-0485-8

Ferrucci, R., Brunoni, A., Parazzini, M., Vergari, M., Rossi, E., Fumagalli, M., et al. (2013). Modulating human procedural learning by cerebellar transcranial direct current stimulation. Cerebellum 12, 485-492. doi: 10.1007/s12311-0120436-9

Frysinger, R. C., Bourbonnais, D., Kalaska, J. F., and Smith, A. M. (1984). Cerebellar cortical activity during antagonist cocontraction and reciprocal inhibition of forearm muscles. J. Neurophysiol. 51, 32-49.

Giannetti, S., and Molinari, M. (2002). Cerebellar input to the posterior parietal cortex in the rat. Brain Res. Bull. 58, 481-489. doi: 10.1016/s03619230(02)00815-8

Grimaldi, G., Argyropoulos, G. P., Boehringer, A., Celnik, P., Edwards, M. J., Ferrucci, R., et al. (2014). Non-invasive cerebellar stimulation-a consensus paper. Cerebellum 13, 121-138. doi: 10.1007/s12311-013-0514-7

Ito, M. (2005). Bases and implications of learning in the cerebellum-adaptive control and internal model mechanism. Prog. Brain Res. 148, 95-109. doi: 10. 1016/s0079-6123(04)48009-1

Ivry, R. (2000). Exploring the role of the cerebellum in sensory anticipation and timing: commentary on Tesche and Karhu. Hum. Brain Mapp. 9, 115-118. doi: 10.1002/(sici)1097-0193(200003)9:3<115::aid-hbm1>3.3.co;2-x 
Izawa, J., Criscimagna-Hemminger, S. E., and Shadmehr, R. (2012). Cerebellar contributions to reach adaptation and learning sensory consequences of action. J. Neurosci. 32, 4230-4239. doi: 10.1523/JNEUROSCI.6353-11.2012

Jankowska, E., Nilsson, E., and Hammar, I. (2011). Processing information related to centrally initiated locomotor and voluntary movements by feline spinocerebellar neurones. J. Physiol. 589, 5709-5725. doi: 10.1113/jphysiol.2011. 213678

Kaelin-Lang, A., Luft, A. R., Sawaki, L., Burstein, A. H., Sohn, Y. H., and Cohen, L. G. (2002). Modulation of human corticomotor excitability by somatosensory input. J. Physiol. 540(Pt. 2), 623-633. doi: 10.1113/jphysiol.2001.012801

Knolle, F., Schrager, E., and Kotz, S. A. (2013). Cerebellar contribution to the prediction of self-initiated sounds. Cortex 49, 2449-2461. doi: 10.1016/j.cortex. 2012.12.012

Kolodziejak, A., Dziduszko, J., Niechaj, A., and Tarnecki, R. (2000). Influence of acute cerebellar lesions on somatosensory evoked potentials (SEPs) in cats. J. Physiol. Pharmacol. 51, 41-55.

Koziol, L., Budding, D., Andreasen, N., D’Arrigo, S., Bulgheroni, S., Imamizu, H., et al. (2014). Consensus paper: the cerebellum's role in movement and cognition. Cerebellum 13, 151-177. doi: 10.1007/s12311-013-0511-x

Leggio, M. G., Chiricozzi, F. R., Clausi, S., Tedesco, A. M., and Molinari, M. (2011). The neuropsychological profile of cerebellar damage: the sequencing hypothesis. Cortex 47, 137-144. doi: 10.1016/j.cortex.2009.08.011

Leggio, M. G., Tedesco, A. M., Chiricozzi, F. R., Clausi, S., Orsini, A., and Molinari, M. (2008). Cognitive sequencing impairment in patients with focal or atrophic cerebellar damage. Brain 131, 1332-1343. doi: 10.1093/brain/awn040

Luft, A. R., Kaelin-Lang, A., Hauser, T. K., Buitrago, M. M., Thakor, N. V., Hanley, D. F., et al. (2002). Modulation of rodent cortical motor excitability by somatosensory input. Exp. Brain Res. 142, 562-569. doi: 10.1007/s00221-0010952-1

Mari, S., Serrao, M., Casali, C., Conte, C., Martino, G., Ranavolo, A., et al. (2014). Lower limb antagonist muscle co-activation and its relationship with gait parameters in cerebellar ataxia. Cerebellum 13, 226-236. doi: 10.1007/s12311013-0533-4

Moberget, T., Karns, C. M., Deouell, L. Y., Lindgren, M., Knight, R. T., and Ivry, R. B. (2008). Detecting violations of sensory expectancies following cerebellar degeneration: a mismatch negativity study. Neuropsychologia 46, 2569-2579. doi: 10.1016/j.neuropsychologia.2008.03.016

Molinari, M., Chiricozzi, F., Clausi, S., Tedesco, A., De Lisa, M., and Leggio, M. (2008). Cerebellum and detection of sequences, from perception to cognition. Cerebellum 7, 611-615. doi: 10.1007/s12311-008-0060-x

Morton, S. M., and Bastian, A. J. (2006). Cerebellar contributions to locomotor adaptations during splitbelt treadmill walking. J. Neurosci. 26, 9107-9116. doi: 10.1523/jneurosci.2622-06.2006

Nixon, P. D. (2003). The role of the cerebellum in preparing responses to predictable sensory events. Cerebellum 2, 114-122. doi: 10.1080/147342203 09410

Oulad Ben Taib, N., and Manto, M. (2008). Reinstating the ability of the motor cortex to modulate cutaneomuscular reflexes in hemicerebellectomized rats. Brain Res. 1204, 59-68. doi: 10.1016/j.brainres.2008.02.014
Popa, L. S., Hewitt, A. L., and Ebner, T. J. (2012). Predictive and feedback performance errors are signaled in the simple spike discharge of individual purkinje cells. J. Neurosci. 32, 15345-15358. doi: 10.1523/JNEUROSCI.2151-12. 2012

Restuccia, D., Della, M. G., Valeriani, M., Leggio, M. G., and Molinari, M. (2007). Cerebellar damage impairs detection of somatosensory input changes. A somatosensory mismatch-negativity study. Brain 130(Pt. 1), 276-287. doi: 10. 1093/brain/awl236

Restuccia, D., Valeriani, M., Barba, C., Le Pera, D., Capecci, M., Filippini, V., et al. (2001). Functional changes of the primary somatosensory cortex in patients with unilateral cerebellar lesions. Brain 124(Pt. 4), 757-768. doi: 10 1093/brain/124.4.757

Schmahmann, J. D., and Pandya, D. N. (1989). Anatomical investigation of projections to the basis pontis from posterior parietal association cortices in rhesus monkey. J. Comp. Neurol. 289, 53-73. doi: 10.1002/cne.902890105

Shadmehr, R., Smith, M. A., and Krakauer, J. W. (2010). Error correction, sensory prediction and adaptation in motor control. Annu. Rev. Neurosci. 33, 89-108. doi: 10.1146/annurev-neuro-060909-153135

Sirigu, A., Cohen, L., Zalla, T., Pradat-Diehl, P., Van Eeckhout, P., Grafman, J., et al. (1998). Distinct frontal regions for processing sentence syntax and story grammar. Cortex 34, 771-778. doi: 10.1016/s0010-9452(08)70780-9

Sotelo, C. (2008). Viewing the cerebellum through the eyes of Ramón Y Cajal. Cerebellum 7, 517-522. doi: 10.1007/s12311-008-0078-0

Takakusaki, K. (2013). Neurophysiology of gait: from the spinal cord to the frontal lobe. Mov. Disord. 28, 1483-1491. doi: 10.1002/mds.25669

Tedesco, A. M., Chiricozzi, F. R., Clausi, S., Lupo, M., Molinari, M., and Leggio, M. G. (2011). The cerebellar cognitive profile. Brain 134, 3672-3678. doi: 10. 1093/brain/awr266

Tesche, C. D., and Karhu, J. J. (2000). Anticipatory cerebellar responses during somatosensory omission in man. Hum. Brain Mapp. 9, 119-142. doi: 10. 1002/(sici)1097-0193(200003)9:3 < 119::aid-hbm2>3.3.co;2-i

Topka, H., Konczak, J., Schneider, K., Boose, A., and Dichgans, J. (1998). Multijoint arm movements in cerebellar ataxia. Exp. Brain Res. 119, 493-503. doi: 10. $1007 /$ s002210050365

Conflict of Interest Statement: The authors declare that the research was conducted in the absence of any commercial or financial relationships that could be construed as a potential conflict of interest.

Received: 26 February 2014; accepted: 10 June 2014; published online: 25 June 2014. Citation: Pisotta I and Molinari M (2014) Cerebellar contribution to feedforward control of locomotion. Front. Hum. Neurosci. 8:475. doi: 10.3389/fnhum.2014.00475 This article was submitted to the journal Frontiers in Human Neuroscience. Copyright (C) 2014 Pisotta and Molinari. This is an open-access article distributed under the terms of the Creative Commons Attribution License (CC BY). The use, distribution or reproduction in other forums is permitted, provided the original author(s) or licensor are credited and that the original publication in this journal is cited, in accordance with accepted academic practice. No use, distribution or reproduction is permitted which does not comply with these terms. 